\title{
Previsão de Ocorrência de Picos Populacionais de Percevejos Pragas da Soja em Condições de Campo
}

\author{
Francisco J. Cividanes $^{1}$ e Jairo G. Figueiredo ${ }^{1}$ \\ ${ }^{1}$ Departamento de Entomologia e Nematologia, FCAV-UNESP, \\ 14870-000, Jaboticabal, SP.
}

\begin{abstract}
An. Soc. Entomol. Brasil 26(3): 517-525 (1997)
Predicting Populational Peaks of Soybean Stink Bugs Under Field Conditions

ABSTRACT - The study was carried out to predict, in the field, the occurrence of populational peaks of adults of Piezodorus guildinii (West.), Nezara viridula (L.) and Euschistus heros (Fabr.) (Heteroptera: Pentatomidae), using a degreeday model. Considering the development of the biological cycle, it was observed that more than $70 \%$ of adult emergence of $P$. guildinii, $N$. viridula and $E$. heros occurred when means of 307.7, 753.8 and 370.3 degree-day respectively had been accumulated. The differences between predicted and observed dates for emergence were 0-3 days for $P$. guildinii and $N$. viridula and 5-6 days for $E$. heros.
\end{abstract}

KEY WORDS: Insecta, Piezodorus guildinii, Nezara viridula, Euschistus heros, Glycine max, degree-day.

RESUMO - Estudou-se em condições de campo, a previsão de picos populacionais de adultos de Piezodorus guildinii (West.), Nezara viridula (L.) e Euschistus heros (Fabr.) (Heteroptera: Pentatomidae), por meio de um modelo de graus-dia. Considerando-se o desenvolvimento do ciclo biológico, observouse que mais de $70 \%$ da emergência dos adultos de $P$. guildinii, $N$. viridula e $E$. heros ocorreu depois de terem sido acumulados em média 307,7; 753,8 e 370,3 graus-dia respectivamente, sendo as diferenças entre a data prevista e a observada de 0-3 dias para P. guildinii e N. viridula e 5-6 dias E. heros.

PALAVRAS-CHAVE: Insecta, Piezodorus guildinii, Nezara viridula, Euschistus heros, Glycine max, graus-dia.

Modelos de graus-dia têm sido muito utilizados em ecologia e manejo de pragas, sendo importantes para a previsão de ocorrência de pragas e inimigos naturais (Obrycki \& Tauber 1981, Braman et al. 1992, Bernal \& González 1993), construção de modelos de simulação por computador (Higley et al. 1986) e para o entendimento de interações ecológicas de pragas e inimigos naturais (Raffa et al. 1992, Thireau \& Regniere 1995).

Entre os percevejos pragas da soja, Glycine max (L.) Merrill, as espécies Piezodorus guildinii (West.), Nezara viridula (L.) e Euschistus heros (Fabr.) (Heteroptera: Pentatomidae), são considerados os mais freqüentes no Brasil (Villas Boâs et al. 1985). Esses percevejos tiveram suas exigências 
térmicas determinadas em laboratório por Cividanes \& Parra (1994a,b,c), e o objetivo deste trabalho foi verificar a nível de campo, a acuracidade da previsão da ocorrência de picos populacionais de adultos destes percevejos por meio de um modelo de grausdia.

\section{Material e Métodos}

O trabalho foi desenvolvido no Câmpus da FCAV-UNESP, Jaboticabal, SP, em uma área aproximada de $2000 \mathrm{~m}^{2}$. No plantio da soja o solo foi adubado com $160 \mathrm{~kg} / \mathrm{ha}$ da fórmula 2:30:10, sendo utilizadas as cultivares de soja IAC-11, Dourados e Foscarim no estudo de $P$. guildinii, $N$. viridula e E. heros, respectivamente.

As posturas dos percevejos foram obtidas de adultos coletados em campos de soja localizados em Guaíra e Jaboticabal. Estes adultos foram mantidos em gaiolas construídas baseando-se em Corrêa-Ferreira (1985) e alimentados com plantas de soja com vagens verdes e grãos secos de soja e amendoim, tendo a água sido fornecida por meio de algodão umedecido diariamente.

O acompanhamento do desenvolvimento dos percevejos nos campos de soja foi feito em duas gaiolas construídas com tela branca e dimensões de $2 \times 2 \times 2 \mathrm{~m}$. Em cada gaiola, quando a soja encontrava-se no estádio de formação da semente (Fehr \& Caviness 1977), cerca de 650 ovos do dia, coletados entre 8:00-9:00 horas e da espécie a ser estudada, foram introduzidos nas mesmas. As posturas foram presas a uma pequena placa de isopor por meio de um alfinete, que perfurou um dos ovos da postura. A seguir, a placa foi fixada na tela de um dos lados da gaiola, ficando localizada abaixo do topo das plantas. Ao ocorrer a eclosão das ninfas, estas foram colocadas nas vagens das plantas de soja, e passaram a ser observadas para o registro do número de adultos emergidos. As observações da eclosão das ninfas e da emergência dos adultos foram feitas diariamente entre 17:0018:00 horas. No caso do N. viridula, as posturas foram obtidas junto ao Centro
Nacional de Pesquisa de Soja, EMBRAPA, Londrina, PR. Elas foram coletadas entre 9:00-10:00 horas, acondicionadas em gerbox com papel filtro umedecido e transportadas para Jaboticabal, sendo levadas ao campo por volta das 16 horas do mesmo dia.

A soma do número de graus-dias foi feita por meio do método da triangulação simples, utilizando-se as temperaturas máximas e mínimas diárias (Silveira Neto et al. 1976, Bernal \& González 1993), e os valores dos limites térmicos inferiores de desenvolvimento das espécies de percevejos (Cividanes \& Parra 1994a,b,c). As temperaturas utilizadas foram registradas na Estação Agroclimatológica da FCAV-UNESP, situada cerca de $700 \mathrm{~m}$ da área experimental.

\section{Resultados e Discussão}

Comparando-se os períodos de eclosão das ninfas e emergência dos adultos de $P$. guildinii , $N$. viridula e $E$. heros, observa-se pouca variação entre eles. No geral, o total de eclosão/emergência ocorreu após 2-3/5-7 dias do seu início (Figs. 1, 2 e 3). Portanto, o período de incubação foi variável em cada uma das espécies, apesar de terem sido usadas massas de ovos depositadas no mesmo dia, fato também determinado em $N$. viridula por Vélez (1974) e Harris \& Todd (1980b). A variação observada entre o ritmo de emergência dos percevejos estudados se deveu provavelmente, ao fato de serem espécies diferentes de insetos e a biologia destes pentatomídeos poder ser afetada por inúmeros fatores, conforme observado em $N$. viridula por Ali \& Ewiess (1977), Harris \& Todd (1980a, b), Panizzi \& Meneguim (1989) (Figs. 1, 2 e 3).

A menor acuracidade da previsão das fases de desenvolvimento de $P$. guildinii, $N$. viridula e E. heros foi observada no período de incubação, ocorrendo uma diferença de 1-2 dias entre a data prevista e a observada no campo, que necessitou para o desenvolvimento de mais de $70 \%$ dos ovos, de um acúmulo médio de 65,8 graus-dia para $P$. guildinii, 98,5 graus-dia para $N$. viridula e 

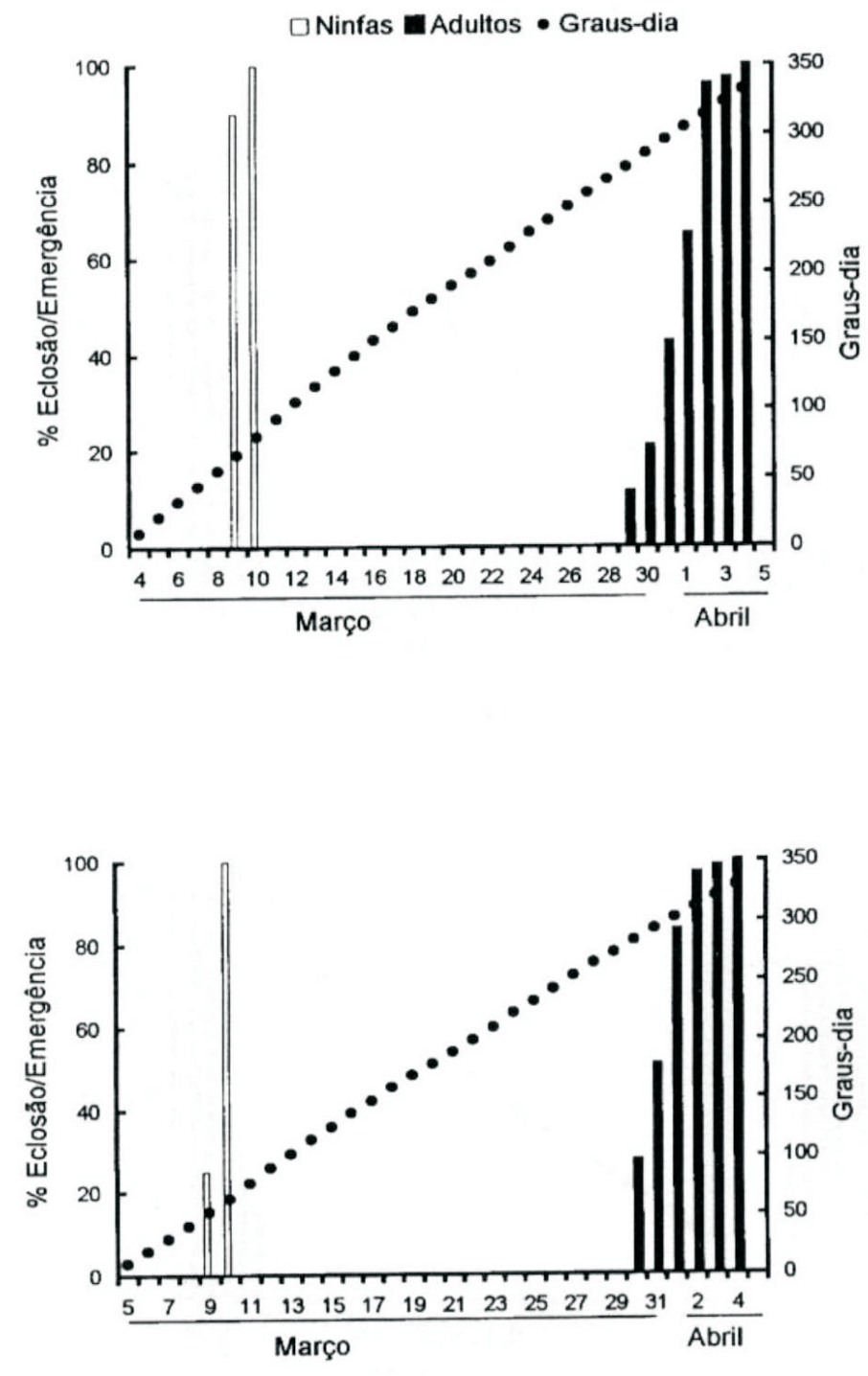

Figura 1. Relação entre a percentagem acumulada de eclosão de ninfas/emergência de adultos de Piezodorus guildinii e a acumulação de graus-dia. Jaboticabal, SP, 1993.

87,8 graus-dia para E. heros (Tabelas 1, 2 e 3). Os fatores que podem ter contribuído para a acuracidade encontrada devem estar relacionados a erros que ocorrem na estimativa do limiar térmico inferior de desenvolvimento e constante térmica nos experimentos de laboratório (Stinner et al. 1974, Bernal \& González 1993), e ao fato das posturas dos percevejos terem sido deixadas em placas de isopor fixadas na tela da gaiola, o que pode ter proporcionado a elas condições térmicas diferente do que se estivessem 

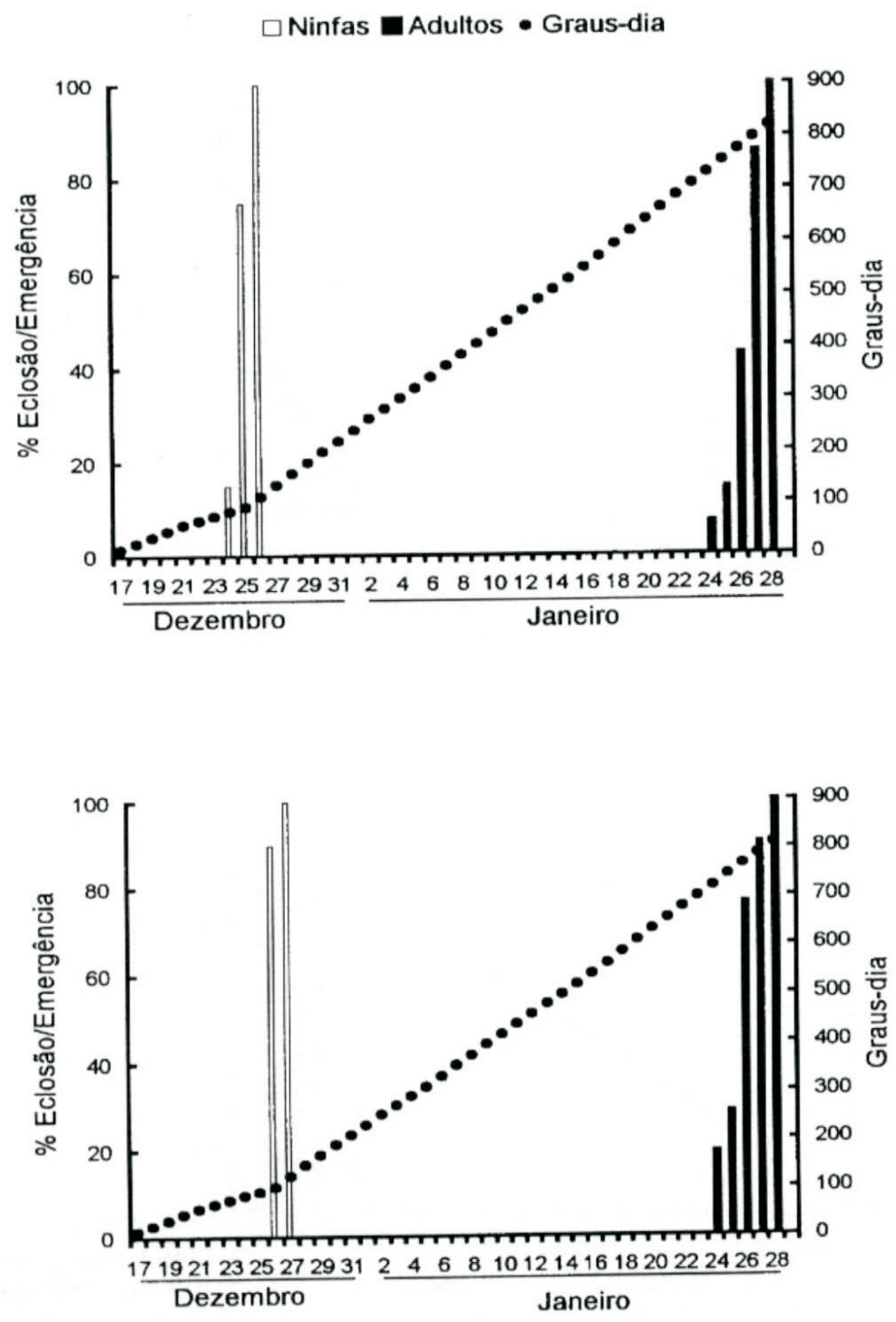

Figura 2. Relação entre a percentagem acumulada de eclosão de ninfas/emergência de adultos de Nezara viridula e a acumulação de graus-dia. Jaboticabal, SP, 1993/94.

espalhadas pelas plantas.

As diferenças observadas entre o acúmulo de graus-dia para as fases de ovo e de ninfa de $N$. viridula com relação a do ciclo biológico (Tabela 2), se deveu ao ajuste do método da hipérbole usado no cálculo das suas exigências térmicas (Cividanes \& Parra 1994a). Quanto ao acúmulo de graus-dia, mais de $70 \%$ das ninfas atingiram a fase adulta quando foram acumulados em média 242,0 graus-dia para $P$. guildinii, 681,9 graus-dia para $N$. viridula e 282,5 graus-dia para $E$. 

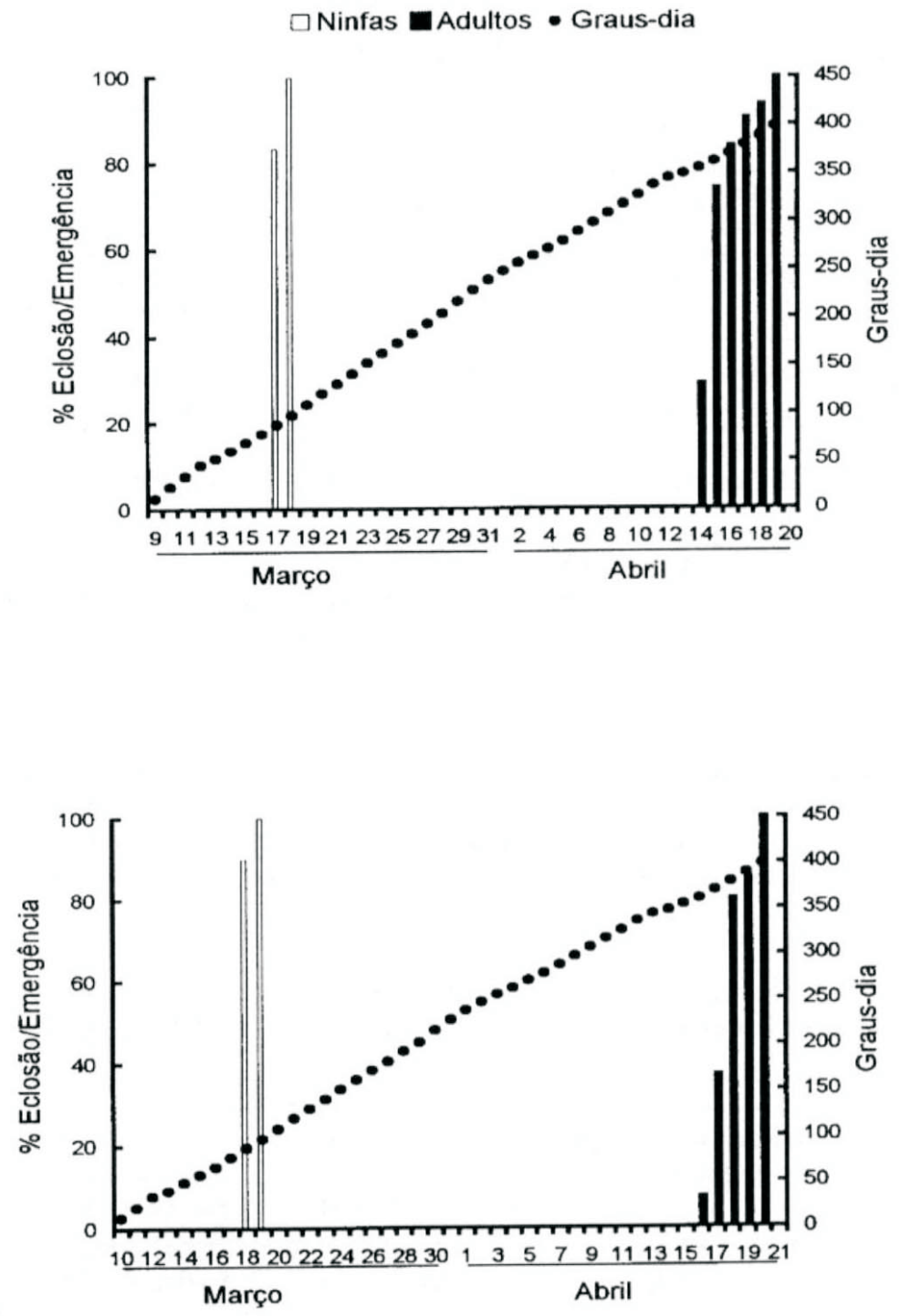

Figura 3. Relação entre a percentagem acumulada de eclosão de ninfas/emergência de adultos de Euschistus heros e a acumulação de graus-dia. Jaboticabal, SP, 1995.

heros (Tabelas 1, 2 e 3). Com relação ao ciclo biológico (ovo-adulto), mais de $70 \%$ da emergência foi verificada com a acumulação média de 307,7; 753,8 e 370,3 graus-dia para P. guildinii, N. viridula e E. heros, respectivamente. Para as três espécies, a ocorrência prevista e observada da fase de ninfa foi de 1-2 dias de diferença. Já para o ciclo biológico, esta diferença variou de 0-3 dias para $P$. guildinii e $N$. viridula e de 5-6 dias para E. heros (Tabelas 1, 2 e 3). Os resultados obtidos para $E$. heros podem ter 
Tabela 1. Ocorrência prevista e observada dos períodos de incubação, desenvolvimento das ninfas e ciclo biológico (ovo-adulto) de Piezodorus guildinii. Jaboticabal, SP, 1993.

\begin{tabular}{|c|c|c|c|c|c|}
\hline \multirow{2}{*}{ Fase } & \multirow{2}{*}{\multicolumn{2}{|c|}{$\begin{array}{c}\text { Data } \\
\text { (início acumulação } \\
\text { graus-dia) }\end{array}$}} & \multicolumn{2}{|c|}{ Ocorrência } & \multirow{2}{*}{$\begin{array}{c}\% \text { Erro } \\
(\mathrm{E})^{3}\end{array}$} \\
\hline & & & $\begin{array}{c}\text { Prevista } \\
{\text { (constante térmica })^{1}}^{\text {(c) }}\end{array}$ & $\begin{array}{c}\text { Observada }^{2} \\
\text { (graus-dia acumulados) }\end{array}$ & \\
\hline \multirow[t]{2}{*}{ Ovo } & $\mathrm{A}^{4}$ & 4 mar. & 8 mar. $(50,6)$ & 9 mar. $(67,2)$ & 32,8 \\
\hline & B & 5 mar. & 9 mar. & 10 mar. $(64,3)$ & 27,1 \\
\hline \multirow[t]{2}{*}{ Ninfa } & A & 10 mar. & 1 abr. $(236,9)$ & 2 abr. $(246,5)$ & 4,1 \\
\hline & $\mathrm{B}$ & 11 mar. & 3 abr. & 1 abr. $(237,4)$ & 0,0 \\
\hline Ciclo bio- & A & 4 mar. & 30 mar. $(288,3)$ & 2 abr. $(313,7)$ & 8,8 \\
\hline lógico & B & 5 mar. & 1 abr. & 1 abr. $(301,7)$ & 4,6 \\
\hline
\end{tabular}

${ }^{1}$ Dados de Cividanes \& Parra 1994 c.

${ }^{2}$ Ocorrência acima de $70 \%$ de eclosão das ninfas ou de emergência de adultos.

${ }^{3} \mathrm{E}=(\mathrm{GDA}-\mathrm{K}) / \mathrm{K}$, onde GDA = graus-dia acumulados, $\mathrm{K}=$ constante térmica.

${ }^{4}$ Gaiolas A e B.

sido em parte causados pelo fato de ser ele uma espécie mais adaptada a regiões onde predominam temperaturas elevadas (Cividanes \& Parra 1994b), e o experimento ter sido desenvolvido durante um período em que as temperaturas estavam em declínio (março-abril). A média das temperaturas mínimas registradas no experimento com $E$.

Tabela 2. Ocorrência prevista e observada dos períodos de incubação, desenvolvimento das ninfas e ciclo biológico (ovo-adulto) de Nezara viridula. Jaboticabal, SP, 1993/94.

\begin{tabular}{|c|c|c|c|c|c|}
\hline \multirow{2}{*}{ Fase } & \multirow{2}{*}{\multicolumn{2}{|c|}{$\begin{array}{l}\text { Data } \\
\text { (início acumulação } \\
\text { graus-dia) }\end{array}$}} & \multicolumn{2}{|c|}{ Ocorrência } & \multirow[b]{2}{*}{$\begin{array}{l}\% \text { Erro } \\
(\mathrm{E})^{3}\end{array}$} \\
\hline & & & $\begin{array}{c}\text { Prevista } \\
\text { (constante térmica) }^{1}\end{array}$ & $\begin{array}{c}\text { Observada }^{2} \\
\text { (graus-dia acumulados) }\end{array}$ & \\
\hline \multirow[t]{2}{*}{ Ovo } & $\mathrm{A}^{4}$ & $17 \mathrm{dez}$. & 24 dez. $(80,8)$ & 25 dez. $(93,2)$ & 15,3 \\
\hline & B & $17 \mathrm{dez}$ & 24 dez. & 26 dez. $(103,8)$ & 28,5 \\
\hline \multirow[t]{2}{*}{ Ninfa } & A & 26 dez. & 28 dez. $(704,2)$ & 27 jan. $(702,8)$ & 0,2 \\
\hline & B & $27 \mathrm{dez}$ & $28 \mathrm{dez}$ & 26 jan. $(661,0)$ & 6,1 \\
\hline \multirow{2}{*}{$\begin{array}{l}\text { Ciclo bio- } \\
\text { lógico }\end{array}$} & A & $17 \mathrm{dez}$. & 26 jan. $(741,0)$ & 27 jan. $(763,0)$ & 3,0 \\
\hline & $\mathrm{B}$ & 17 dez. & 26 jan. & 26 jan. $(744,5)$ & 0,4 \\
\hline
\end{tabular}

${ }^{1}$ Dados de Cividanes \& Parra 1994 a.

${ }^{2}$ Ocorrência acima de $70 \%$ de eclosão das ninfas ou de emergência de adultos.

${ }^{3} \mathrm{E}=(\mathrm{GDA}-\mathrm{K}) / \mathrm{K}$, onde GDA = graus-dia acumulados, $\mathrm{K}=$ constante térmica.

${ }^{4}$ Gaiolas A e B. 
heros foi cerca de um grau mais baixa do que as registradas nos experimentos com $P$. guildinii e $N$. viridula, sendo que em dois dias limiares térmicos padrões de 5,10 ou $15^{\circ} \mathrm{C}$

Modelos de graus-dia que façam previsões com $10-15 \%$ de precisão são indicados para

Tabela 3. Ocorrência prevista e observada dos períodos de incubação, desenvolvimento das ninfas e ciclo biológico (ovo-adulto) de Euschistus heros. Jaboticabal, SP, 1995.

\begin{tabular}{|c|c|c|c|c|c|}
\hline \multirow{2}{*}{ Estágio } & \multirow{2}{*}{\multicolumn{2}{|c|}{$\begin{array}{c}\text { Data } \\
\text { (início acumulação - } \\
\text { graus-dia) }\end{array}$}} & \multicolumn{2}{|c|}{ Ocorrência } & \multirow{2}{*}{$\begin{array}{l}\% \text { Erro } \\
(\mathrm{E})^{3}\end{array}$} \\
\hline & & & $\begin{array}{c}\text { Prevista } \\
(\text { constante térmica })^{1}\end{array}$ & $\begin{array}{c}\text { Observada }^{2} \\
\text { (graus-dia acumulados) }\end{array}$ & \\
\hline \multirow[t]{2}{*}{ Ovo } & $\mathrm{A}^{4}$ & 9 mar. & 15 mar. $(66,1)$ & 17 mar. $(87,9)$ & 32,9 \\
\hline & B & 10 mar. & 16 mar. & 18 mar. $(87,7)$ & 32,7 \\
\hline \multirow[t]{2}{*}{ Ninfa } & A & 18 mar. & 13 abr. $(261,8)$ & 15 abr. $(273,7)$ & 4,5 \\
\hline & B & 19 mar. & 17 abr. & 18 abr. $(291,2)$ & 11,2 \\
\hline Ciclo bio- & $\mathrm{A}$ & 9 mar. & 10 abr. $(327,8)$ & 15 abr. $(361,6)$ & 10,3 \\
\hline lógico & $\mathrm{B}$ & 10 mar. & 12 abr. & 18 abr. $(378,9)$ & 15,6 \\
\hline
\end{tabular}

${ }^{1}$ Dados de Cividanes \& Parra 1994 b.

${ }^{2}$ Ocorrência acima de $70 \%$ de eclosão das ninfas ou de emergência de adultos.

${ }^{3} \mathrm{E}=(\mathrm{GDA}-\mathrm{K}) / \mathrm{K}$, onde GDA = graus-dia acumulados, $\mathrm{K}=$ constante térmica.

${ }^{4}$ Gaiolas A e B.

a temperatura mínima foi menor do que o limiar térmico inferior de desenvolvimento de E. heros, fato não ocorrido durante os outros dois experimentos. De qualquer modo, a acuracidade da previsão do desenvolvimento das ninfas e do ciclo biológico (ovo-adulto) foi maior que o da fase de ovo (Tabelas 1, 2 e 3 ). Devendo ser destacado, que os insetos geralmente apresentam limiar térmico diferente em cada fase de desenvolvimento (Sanborn et al. 1982). No entanto, o uso destes valores tem sido simplificado na prática, pois com freqüência utiliza-se um único valor para representar o limiar térmico, obtido pela média dos valores das diferentes fases ou pela estimativa usando-se dados do ciclo biológico ( Higley et al. 1986, Cividanes \& Figueiredo 1996). No futuro, o uso destes valores talvez seja mais simplificado ainda, pois Pruess (1983) recomendou que limiares térmicos com potencial de serem usados em programas de manejo de pragas devem ser ajustados a o manejo integrado de pragas a que se destinam (Higley et al. 1986). Portanto, os resultados obtidos no presente estudo são realísticos, mostrando que o modelo de grausdia utilizado teve boa acuracidade, podendo ser usado para a previsão de picos populacionais de adultos de $P$. guildinii, $N$. viridula e E. heros em programas de manejo de pragas da soja.

\section{Agradecimentos}

Os autores agradecem à Dra. Beatriz S. Corrêa-Ferreira da Embrapa Soja, por ter gentilmente cedido as posturas de Nezara viridula usadas neste estudo.

\section{Literatura Citada}

Ali, M. \& M.A. Eweiss. 1977. Photoperiodic and temperature effects on rate of development and diapause in the green 
stink bug, Nezara viridula (L.) (Heteroptera: Pentatomidae). Acta Phytoph. Acad. Sci. Hung. 12:357-347.

Bernal, J. \& D. González. 1993. Experimental assessment of a degree-day model for predicting the development of parasites in the field. J. Appl. Entomol. 116:459-466.

Braman, S.K., A. F. Pendley, B. Sparks \& W. G. Hudson. 1992. Thermal requirements for development, population trends, and parasitism of azalea lace bug (Heteroptera: Tingidae). J. Econ. Entomol. 85: 870-877.

Cividanes, F.J. \& J. R. P. Parra. 1994a. Biologia em diferentes temperaturas e exigências térmicas de percevejos pragas da soja. I. Nezara viridula (L.) (Heteroptera:Pentatomidae). An Soc. Entomol. Brasil 23:243-250.

Cividanes, F.J. \& J. R. P. Parra. 1994b. Biologia em diferentes temperaturas e exigências térmicas de percevejos pragas da soja. II. Euschistus heros (Fabr.) (Heteroptera:Pentatomidae). Pesq. Agropec. Bras. 29: 1841-1846.

Cividanes, F.J. \& J. R. P. Parra. 1994c. Biologia em diferentes temperaturas e exigências térmicas de percevejos pragas da soja. III. Piezodorus guildinii (West.) (Heteroptera:Pentatomidae). Científica 22:177-186.

Cividanes, F.J. \& J.G. Figueiredo. 1996. Desenvolvimento e emergência de Trissolcus brochymenae (Ashmead) e Telenomus podisi Ashmead (Hymenoptera: Scelionidae) em diferentes temperaturas. An. Soc. Entomol. Brasil 25:207211.

Harris, V.E. \& J.W. Todd. 1980a. Comparative fecundity, egg fertility and hatch among three laboratory-reared generations of the southern green stink bug Nezara viridula (L.) (Hemiptera: Pentatomidae). J. Georgia Entomol. Soc. 15:245-253.

Harris, V.E. \& J.W. Todd. 1980b. Duration of immature stages of the southern green stink bug, Nezara viridula (L.) with a comparative review of previous studies. J. Geogia Entomol. Soc. 15:114-124.

Corrêa-Ferreira, B.S. 1985. Criação massal do percevejo verde Nezara viridula (L.). Londrina, EMBRAPA-CNPSo, Doc. 11, $16 \mathrm{p}$.

Fehr, W. R. \& C. E. Caviness. 1977. Stages of soybean development. Ames, Coop. Ext. Serv., Iowa State University, Special Report 80, 12p.

Higley, L.G., L. P. Pedigo \& K. R. Ostlie. 1986. DEGDAY: a program for calculating degree-days, and assumption behind the degree-day approach. Environ. Entomol. 15:999-1016.

Obrycki, J.J. \& M. J. Tauber. 1981. Phenology of three coccinellid species: thermal requirements for development. An. Entomol. Soc. Am. 74:31-36.

Panizzi, A.R. \& A.M. Meneguim. 1989. Performance of ninphal and adult Nezara viridula on selected alternate host plants. Entomol. Exp. Appl. 50:215-223.

Pruess, K.P. 1983. Day-degree methods for pest management. Environ. Entomol. 12:613-619.

Raffa, K. F., D. J. Hall, W. Kearby \& S. Katovich. 1992. Seasonal life history of introduced basswood thrips (Thysanoptera: Thripidae) in Wisconsin, with observations on associated thrips species. Environ. Entomol. 21:771-779.

Sanborn, S.M., J.A. Wyman \& A.J. 
Mueller. 1982. Threshold temperature and heat unit summations for seedcorn maggot development under controlled conditions. An. Entomol. Soc. Am. 75:399-401.

\section{Silveira Neto, S., O. Nakano, D. Barbin \&} N. A. Villa Nova. 1976. Manual de ecologia dos insetos. São Paulo, Ceres, 419p.

Stinner, R. E., A. P. Guttierrez \& G. D. Butler Jr. 1974. An algorithm for temperature-dependent growth rate simulation. Can. Entomol. 106:519-524. trachynotus with its hosts Choristoneura fumiferana and C. rosaceana. Entomol. Exp. Appl. 76:67-82.

Villas Bôas, G. L., F. Moscardi, B. S. Corrêa-Ferreira, C. B. H. Campo, I. C. Corso \& A. R. Panizzi. 1985. Indicações do manejo de pragas para percevejos. Londrina EMBRAPACNPSo, Doc. 9, 15p.

Vélez, J.R. 1974. Observaciones sobre la biologia de la chinche verde, Nezara viridula (L.), en el Valle del Fuerte Sin. Folia Entomol. Mex. 28:5-12.

Thireau, J. C. \& J. Regniere. 1995. Development, reproduction, voltinism and host synchrony of Meteorys

Recebido em 07/04/97. Aceito em 10/10/97. 